\title{
Comparación Antropométrica entre Mandíbulas de las Poblaciones Uruguaya y Brasileña
}

\author{
Anthropometric Comparison between Mandibles from Uruguayan and Brazilian Populations \\ ${ }^{* * * *}$ Carlos Sassi; ${ }^{*, * *}$ Alicia Picapedra; ${ }^{* * *}$ Paulo Henrique Ferreira Caria; ${ }^{* * * *}$ Francisco Groppo; ${ }^{* * * * * * * * *}$ Luiz Francesquini Júnior; \\ ${ }^{* * * * * * *}$ Eduardo Daruge Júnior $\&{ }^{* * * * *}$ Felippe Bevilacqua Prado
}

SASSI, C.; PICAPEDRA, A.; CARIA, P. H. F.; GROPPO, F.; FRANCESQUINI JÚNIOR, L.; DARUGE JÚNIOR, E. \& PRADO

F. B. Comparación antropométrica entre mandíbulas de las poblaciones uruguaya y brasileña. Int. J. Morphol., 30(2):379-387, 2012.

RESUMEN: Las mandíbulas humanas pertenecientes a la población uruguaya presentan ciertas diferencias anatómicas con las de la brasileña. Tal hecho parecería deberse a las particularidades raciales y étnicas de cada país. Este estudio examinó las características morfológicas de 100 mandíbulas de uruguayos y las cotejó con las correspondientes a idéntico número de piezas óseas de brasileños, por medio de trece mediciones estipuladas por Humphrey et al. (1999). Las variaciones significativas entre los valores promedios fueron determinadas mediante el test de Mann-Whitney (alfa=5\%). Se constataron expresivas discrepancias entre algunas de las dimensiones analizadas, producto de las modificaciones de forma y estructura de las mandíbulas de ambos grupos. Las uruguayas exhibieron guarismos clara o ligeramente superiores en prácticamente todas las medidas realizadas, salvo en lo que respecta a la altura de la sínfisis (sutilmente mayores en sus homólogas brasileñas). Las divergencias más evidentes correspondieron a la altura de la incisura, altura de la rama y longitud mandibular, y las menos notorias al ancho mandibular, altura de la sínfisis y ancho bicoronoideo. Los factores que pueden haber inducido a las mismas resultan realmente profusos, incluyendo tanto a las adaptaciones funcionales del sistema estomatognático como a las biomecánicas, estimuladas por la diferente consistencia de la dieta, o la propia miscegenación.

PALABRAS CLAVE: Mandíbula; Morfología; Antropometría; Brasil; Uruguay.

\section{INTRODUCCIÓN}

Resulta un hecho incontrastable que, durante mucho tiempo, el hombre ha llevado a cabo denodados esfuerzos en pos de explicar su propio origen y evolución. En efecto, uno de los grandes desvelos de la ciencia ha sido intentar comprender los fenómenos responsables de ese dinámico proceso multifactorial, poniendo al mismo tiempo de manifiesto, las notorias modificaciones que la especie humana ha sufrido a lo largo de millones de años de existencia. Estas variaciones se tornan evidentes en lo que respecta al tamaño y forma del esqueleto en general y de la mandíbula en particular (Humphrey et al., 1999).

La morfometría y en especial la osteometría, parte de la antropología física que se ocupa de las mediciones de los huesos, han contribuido de manera determinante en los procedimientos de evaluación de las mismas, constituyén- dose en herramientas de verdadera y trascendental importancia, para la realización de estudios comparativos entre diferentes grupos poblacionales (Prado \& Caria, 2007).

Así pues, trabajos como el de Kaifu (1997) sirven de sustento para esta aserción, al demostrar que las modificaciones morfológicas mandibulares constatadas en japoneses, eran debidas a la interacción de diversos factores, tales como: miscegenación, apreciable disminución del esfuerzo masticatorio (como consecuencia de los drásticos cambios en la dieta), localización geográfica y condiciones climáticas.

En concordancia con esa postura, Lieberman et al. (2004) sugieren la influencia de elementos genéticos, ambientales y nutricionales (determinados por la evidente alteración de la composición y consistencia de los alimentos),

\footnotetext{
Doctor en Odontología, Facultad de Odontología de la Universidad de la República, Montevideo, Uruguay.

** Especialista en Odontología Legal y Deontología, Facultad de Odontología de Piracicaba, UNICAMP, São Paulo, Brasil.

*** Profesor del área de Anatomía, Facultad de Odontología de Piracicaba - UNICAMP, SP, Brasil.

***** Profesor del área de Farmacología y Anestesiología, Faculdad de Odontología de Piracicaba, UNICAMP, São Paulo, Brasil

****** Profesor de Odontología Legal de la Facultad de Odontología, São Leopoldo Mandic, Campinas, Brasil

******** Profesor de Odontología Legal y Deontología de la Facultad de Odontología de Piracicaba, UNICAMP, São Paulo, Brasil.
} 
en las sucesivas fases del crecimiento y desarrollo facial, evidenciada por las apreciables diferencias de tamaño, forma y estructura de los huesos de la región.

Sin duda alguna, los tejidos blandos y principalmente los músculos, al igual que las fuerzas oclusales, desempeñan un rol fundamental en el control de la forma de la cara y esencialmente de la mandíbula (Hunt, 1998; Zaffaroni Piaggio \& Fiorestti, 2010; Antón et al., 2011).

Por otra parte, América Latina y el Caribe presentan una realidad demográfica particular, caracterizada por un complejo escenario étnico, cultural y social, fruto del intenso proceso de mestizaje de la mayoría de las poblaciones, del cual sólo quedan exentos algunos grupos aislados de amerindios (Hidalgo et al., 2005).

En este contexto, las particularidades morfológicas encontradas en las mandíbulas de brasileños, parecen ser consecuencia de las numerosas razas y etnias que han contribuido en la determinación de los principales rasgos de la actual población de ese país (Ferreira, 1998; Heringer, 2002; Torres, 2002; Prado \& Caria).

Empero, no es menos cierto que Uruguay ha sido tradicionalmente considerado, como el único país sudamericano cuya población descendía exclusivamente de inmigrantes europeos, si bien recientes investigaciones han revelado la innegable contribución genética de los componentes indígena y africano, en la conformación de la "identidad uruguaya" (Sans et al., 2006; Rodríguez \& González, 2010).

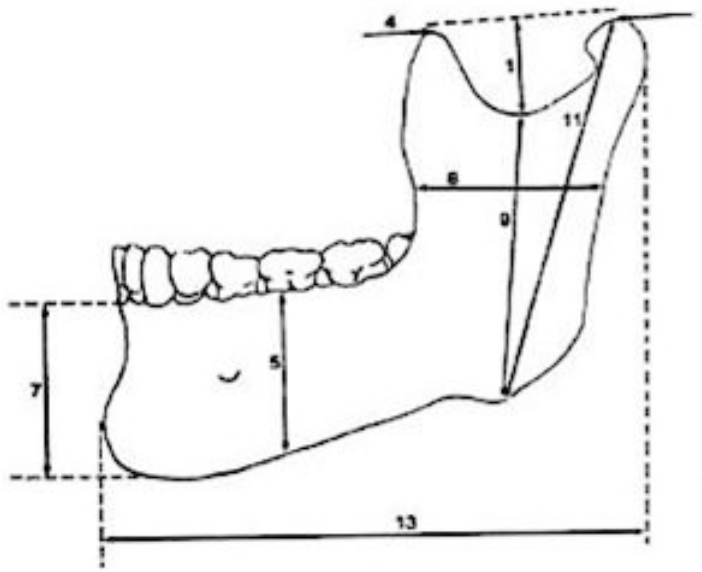

Por todo lo expuesto, el presente trabajo tuvo por objetivo realizar una comparación antropométrica entre mandíbulas de uruguayos y brasileños.

\section{MATERIAL Y MÉTODO}

Se utilizaron 100 mandíbulas humanas de individuos uruguayos, pertenecientes al acervo del Museo de Anatomía de la FO - UDELAR (Facultad de Odontología - Universidad de la República), Montevideo, Uruguay, y 100 de individuos brasileños, del Departamento de Morfología de la FOP UNICAMP (Facultad de Odontología de Piracicaba Universidade de Campinas), São Paulo, Brasil, de las que no se poseía datos que permitiesen asignarles sexo y edad. Fueron seleccionadas aquellas con molares erupcionados, y excluidas las que presentaban anomalías o secuelas de procesos patológicos, intentando establecer puntos de confluencia y concordancia con anteriores trabajos en esta materia, como el de Wood et al. (1991). A seguir, fueron efectuadas trece mediciones en cada una de las mandíbulas, por un único operador, en tres ocasiones diferentes, mediando un intervalo no menor a dos semanas entre cada una de las secuencias respectivas, para evitar recordar los datos ya recabados, cumplir con el correspondiente análisis de confiabilidad y minimizar las posibilidades de cometer errores, con el auxilio de un paquímetro digital de 0,01 mm. de precisión (Digimess ${ }^{\circledR}$, de $300 \mathrm{~mm}$ de capacidad) y un mandibulómetro de Hambruch (modificado por Sassi \& Picapedra), según la metodología descrita por Humphrey et al., basada en trece parámetros morfométricos que incluyen altura, ancho y largo de las referidas piezas óseas (Figs. 1 y 2).

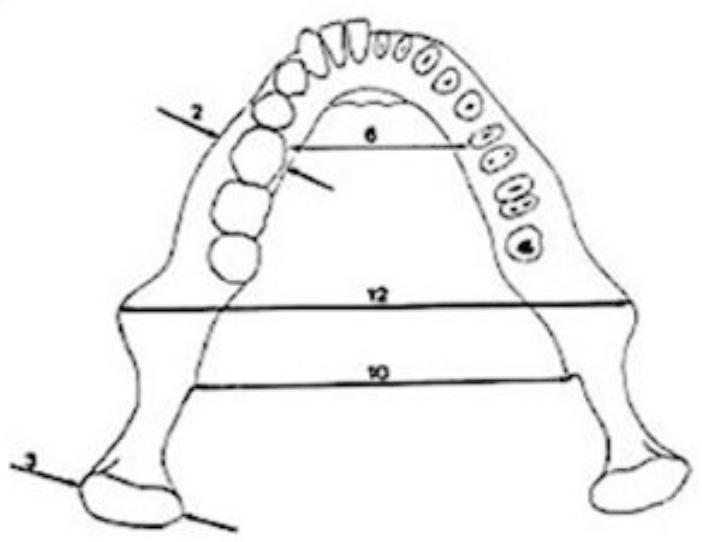

Fig. 1. Ilustración con las trece (13) medidas mandibulares estandarizadas por Humphrey et al. (1999)1. Profundidad de la incisura (escotadura mandibular o sigmoidea). 2. Grosor del cuerpo. 3. Longitud o diámetro mayor del cóndilo (proceso o apófisis condilar). 4. Distancia entre el proceso coronoideo (apófisis coronoides) y el cóndilo. 5. Altura del cuerpo. 6. Ancho mandibular. 7. Altura de la sínfisis. 8. Ancho de la rama. 9. Altura de la incisura (distancia comprendida entre el ángulo antegoníaco y el punto más inferior de la escotadura sigmoidea). 10. Distancia bilingular (entre ambas espinas de Spix). 11. Altura de la rama. 12. Ancho o diámetro bicoronoideo. 13. Longitud mandibular. 


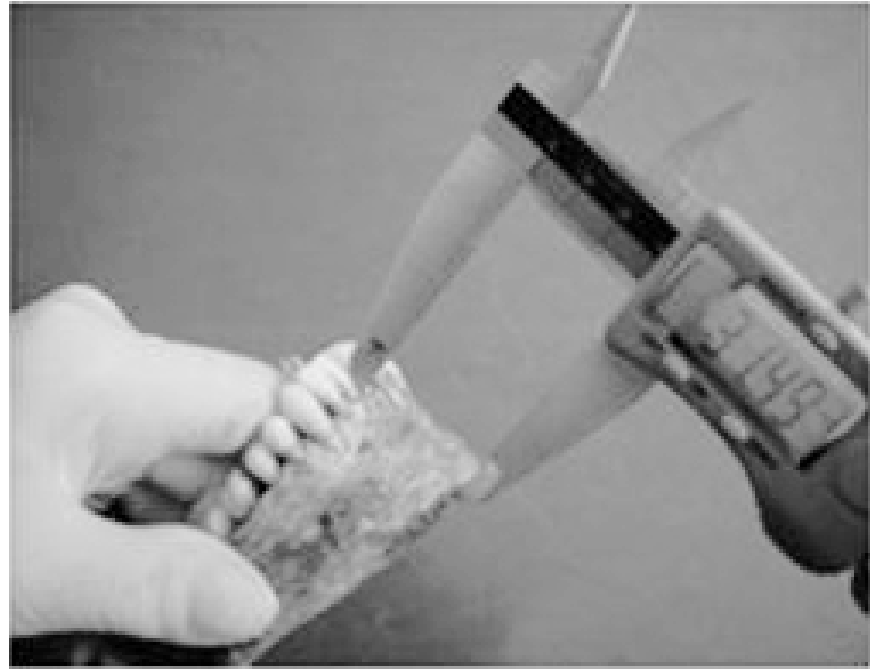

Fig. 2. Instrumentos de medición empleados. Resáltase que el paquímetro digital fue el elegido para consumar las doce (12) primeras medidas enumeradas por Humphrey et al. (1999) y que el mandibulómetro de Hambruch, modificado por Sassi \& Picapedra, resultó de suma utilidad en el caso específico de la longitud mandibular.

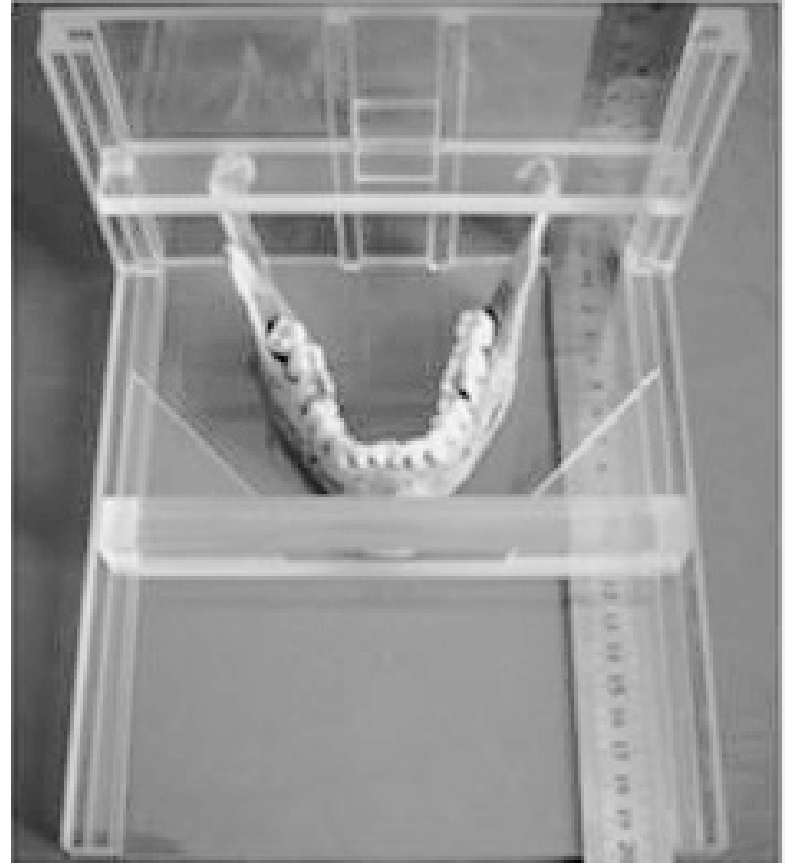

El proyecto fue evaluado por el CEP (Comité de Ética en Pesquisa o Investigación) de la FOP - UNICAMP y el Comité de Ética en Investigación de la FO - UDELAR, dado el carácter binacional de esta iniciativa científica, y finalmente aprobado, según consta en el protocolo de resolución $\mathrm{N}^{\mathrm{o}}$ 010/2006 y expediente 091100-001996-08, respectivamente. pleo del Microsoft Excel y Bioestat 5.0.

Tabla I. Valores promedios ( \pm desvío padrón o estándar) en milímetros (mm) de cada una de las medidas en 100 mandíbulas de individuos brasileños y uruguayos.

\begin{tabular}{lcc}
\hline Mandíbula & Brasileños & Uruguayos \\
\hline Profundidad de la incisura & $13,09( \pm 2,03)$ & $13,82( \pm 2,08)$ \\
Grosor del cuerpo & $11,00( \pm 2,16)$ & $13,52( \pm 1,77)$ \\
Longitud del cóndilo & $18,39( \pm 2,41)$ & $20,09( \pm 2,40)$ \\
Distancia entre el proceso coronoideo y el cóndilo & $32,91( \pm 3,64)$ & $34,68( \pm 3,04)$ \\
Altura del cuerpo & $23,97( \pm 4,12)$ & $25,05( \pm 3,65)$ \\
Ancho mandibular (zona del primer molar) & $36,93( \pm 3,17)$ & $37,65( \pm 3,56)$ \\
Altura de la sínfisis & $29,36( \pm 4,48)$ & $28,18( \pm 3,63)$ \\
Ancho de la rama & $29,30( \pm 4,18)$ & $31,06( \pm 3,10)$ \\
Altura de la incisura & $47,14( \pm 4,55)$ & $50,37( \pm 5,49)$ \\
Distancia bilingular & $78,75( \pm 4,48)$ & $80,17( \pm 4,23)$ \\
Altura de la rama & $64,76( \pm 5,03)$ & $69,01( \pm 6,30)$ \\
Ancho bicoronoideo & $94,71( \pm 6,11)$ & $95,69( \pm 6,28)$ \\
Longitud mandibular & $112,40( \pm 6,42)$ & $117,44( \pm 6,49)$ \\
\hline
\end{tabular}




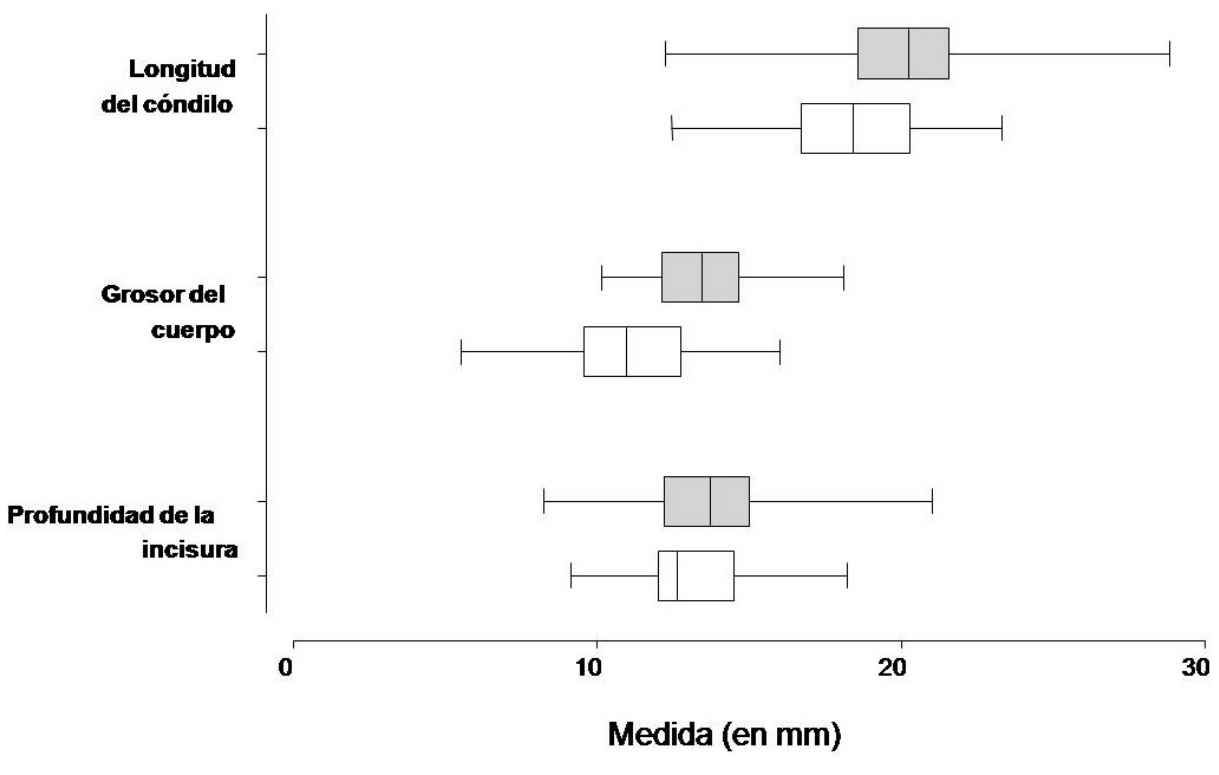

Fig. 3. Profundidad de la incisura, grosor del cuerpo y longitud del cóndilo de las mandíbulas uruguayas (en gris) y brasileñas (en blanco).

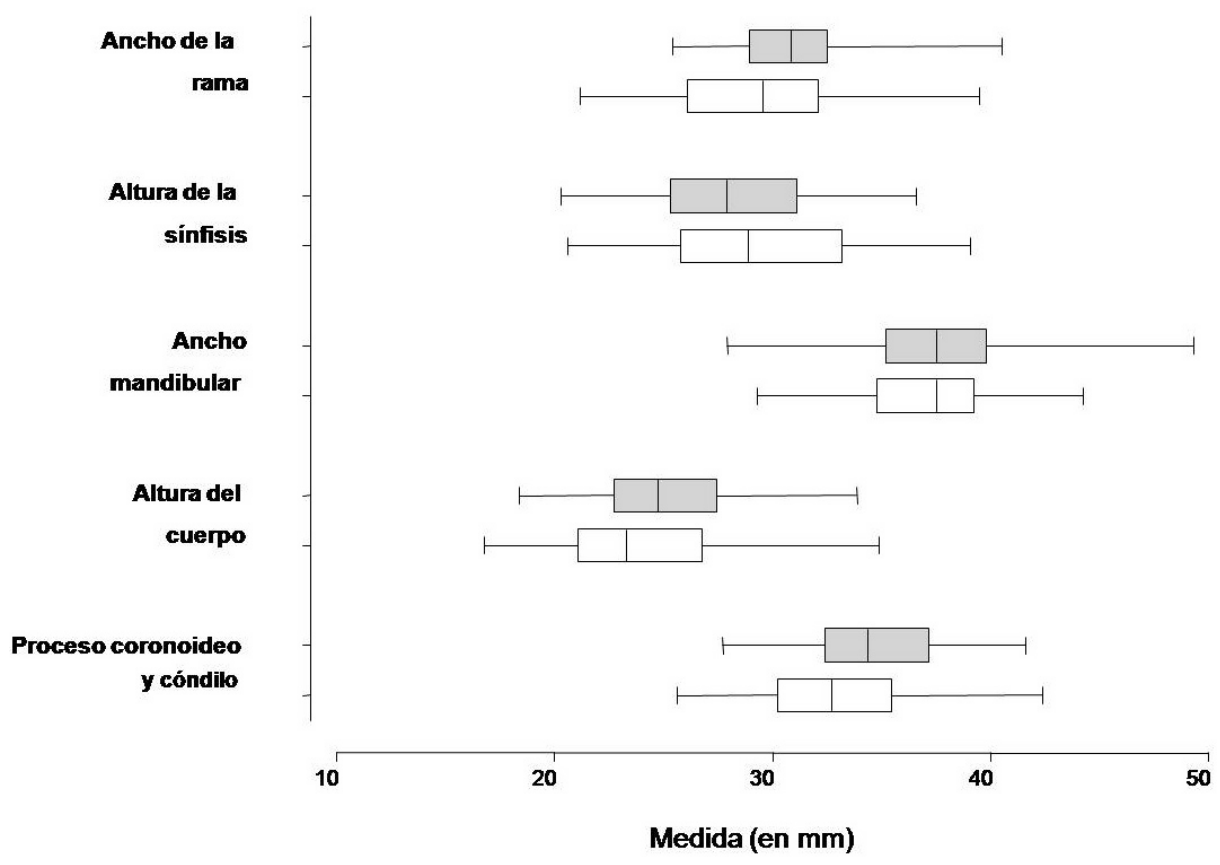

Fig. 4. Distancia entre el proceso coronoideo y el cóndilo, altura del cuerpo, ancho mandibular (zona del primer molar), altura de la sínfisis y ancho de la rama de las mandíbulas uruguayas (en gris) y brasileñas (en blanco).

\section{RESULTADOS}

Los resultados obtenidos fueron analizados detenida y minuciosamente, por medio de la aplicación del coeficiente intraclase, permitiendo establecer un valor de correlación específico $(\mathrm{r}=0,96)$, que revela una concordancia casi total entre las tres series de medidas oportunamente realizadas. Se dejó expresa constancia del desvío de los valores considerados padrón o estándar, así como de los máximos, mínimos y promedios verificados (Tabla I; Figs. 3, 4 y 5).

Por su parte, el test de Mann-Whitney fue empleado para evaluar las potenciales y ostensibles diferencias tipificadas entre las poblaciones consideradas, en lo relativo a los valores medios de cada dimensión. El mismo permitió demostrar que las mandíbulas uruguayas poseían mayor profundidad de la incisura ( $\mathrm{p}=0,0093$ ), grosor del cuerpo $(\mathrm{p}<0,0001)$ y longitud del cóndilo $(\mathrm{p}<0,0001)$ que las brasileñas (Fig. 3).

De manera análoga, fue posible percibir que las mandíbulas uruguayas presentaban valores más altos en lo que respecta a la distancia comprendida entre el proceso coronoideo y el cóndilo $(\mathrm{p}=0,0002)$, altura del cuerpo ( $\mathrm{p}=0,0296)$ y ancho de la rama $(p=0,00039)$. No obstante, no se constataron expresivas discrepancias en relación al ancho mandibular $(\mathrm{p}=0,2009)$, medido en la zona del primer molar, y a la altura de la sínfisis $(\mathrm{p}=0,0674)$, tal como se aprecia en la Fig. 4. 
Finalmente, pudo comprobarse que las mandíbulas uruguayas mostraban una mayor altura de la incisura $(\mathrm{p}<0,0001)$, distancia bilingular $(\mathrm{p}=0,0447)$, altura de la rama $(\mathrm{p}<0,0001)$ y longitud $(\mathrm{p}<0,0001)$. Sin embargo, no hubo divergencias estadísticamente significativas con respecto al ancho bicoronoideo ( $\mathrm{p}=0,2035)$, como se evidencia en la Fig. 5.

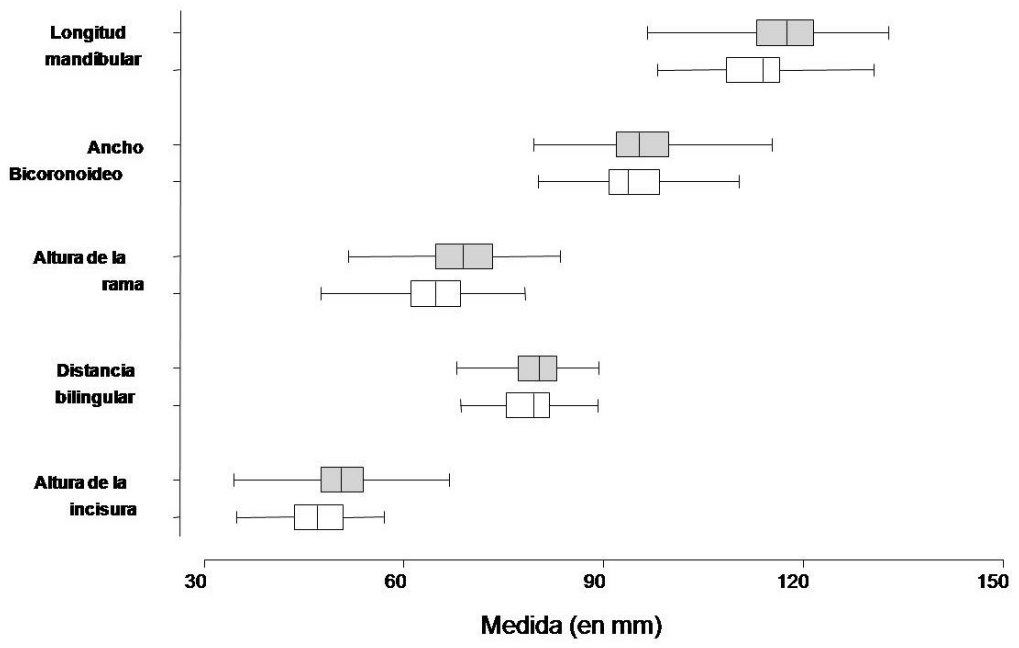

Fig. 5. Altura de la incisura, distancia bilingular, altura de la rama, ancho bicoronoideo y longitud de las mandíbulas uruguayas (en gris) y brasileñas (en blanco).

\section{DISCUSIÓN}

Muchas han sido las investigaciones tendientes a dilucidar y especificar los factores responsables de la hominización y de las modificaciones morfológicas acaecidas en las mandíbulas de los seres humanos, desde su aparición sobre la faz de la tierra hasta hoy. Sucesivos estudios comparativos han puesto de manifiesto que las antedichas se han debido, principalmente, a la influencia directa del ambiente y la genética sobre los dinámicos procesos de crecimiento y remodelación ósea. Del mismo modo, las variaciones suscitadas en los respectivos padrones de reabsorción y neoformación ósea, en conjunción con la cambiante actividad de la musculatura esquelética, pueden haber desempeñado un preponderante papel en ese sentido (Hunt; Humphrey et al.; Bolini et al., 2006; Rai et al., 2007; Zaffaroni Piaggio \& Fiorestti).

Otros autores, como Wood et al., Bogin \& Rios (2003), Suazo et al. (2008) y Antón et al., coinciden en afirmar que las comprobadas alteraciones dimensionales, son producto de una multiplicidad y sucesión de fenómenos que determinan y hasta modifican la función masticatoria y, por ende, la morfología facial de los individuos. De acuerdo con sus experiencias, diversos elementos pueden ser señalados en mayor o menor medida, como agentes etiológicos primordiales o coadyuvantes, en este complejo "escenario biológico", a saber: forma y tamaño del neurocráneo, posición de la cabeza, biotipo facial, determinantes genéticos, características étnicas, potencia muscular, hábitos alimenticios, tamaño de los dientes, factores ambientales, geográficos, climáticos y socioeconómicos, entre otros.

En la presente comparación antropométrica, las variaciones más palmarias se patentizaron en las dimensiones de la altura de la incisura, altura de la rama y longitud mandibular, donde las piezas óseas uruguayas exhibieron guarismos ciertamente superiores $(50,37 \mathrm{~mm} \pm 5,49 \mathrm{~mm}, 69,01 \mathrm{~mm} \pm$ $6,30 \mathrm{~mm}$ y $117,44 \mathrm{~mm} \pm 6,49 \mathrm{~mm})$ a las de sus homólogas brasileñas $(47,14 \mathrm{~mm} \pm 4,55$ $\mathrm{mm}, 64,76 \mathrm{~mm} \pm 5,03 \mathrm{~mm}$ y $112,40 \mathrm{~mm} \pm$ $6,42 \mathrm{~mm}$, respectivamente). Dichas diferencias morfológicas pueden ser fruto de las variadas razas existentes en nuestro planeta, tal cual se desprende de los estudios científicos llevados a cabo por Kaifu y Anderson (1998). A este respecto, el primero, trabajando con mandíbulas de orientales, fósiles y del período moderno, concluyó que la altura de la sínfisis era la dimensión más constante a través de los miles de años transcurridos, mientras que el segundo encontró variaciones sólo en el ancho y altura de la rama mandibular, entre poblaciones de disímiles regiones y estirpes.

Vale la pena resaltar que, por más de tres siglos, tuvo lugar una intensa corriente migratoria, en especial de los continentes europeo, africano y asiático al americano, lo que, sin lugar a dudas, repercutió directamente sobre los aspectos morfológicos, culturales y sociales de los pueblos uruguayo y brasileño.

En el caso de Uruguay, puede aseverarse que durante el período colonial, recibió inmigrantes de ascendencia española, portuguesa y africana, los cuales, conjuntamente con las tribus autóctonas, contribuyeron a poblar la superficie territorial de la Banda Oriental. Antes de que estas últimas fueran exterminadas, los hombres colonizadores interactuaron y convivieron con las mujeres indígenas y las propias africanas, dando comienzo así al proceso de miscegenación de la población (Hidalgo et al.).

Más tarde, en la segunda mitad del siglo XIX, algunos Estados europeos establecieron la "libertad de emigrar" en sus propias Constituciones, confluyendo con la aplica- 
ción de una franca política de "fronteras abiertas" por parte de muchos gobiernos americanos, acontecimientos que resultaron determinantes, para que importantes contingentes se desplazaran desde el viejo hacia el nuevo continente (Sans et al., 1997; Ferreira; Sanguinet, 2001; Heringer; Torres; Prado \& Caria).

El incesante y creciente arribo de extranjeros a Uruguay continuó durante los últimos años del siglo XIX y primera mitad del XX, principalmente provenientes de España e Italia, además de un relevante número de judíos. Quizás ese haya sido el motivo de que se considerase al uruguayo como el más europeo de los pueblos americanos. Sin embargo, investigaciones focalizadas en el estudio de los rasgos morfológicos, sanguíneos y moleculares, han demostrado la incuestionable trascendencia de las vertientes indígena y africana, en la determinación de las raíces genéticas de los uruguayos (Sans, 2000; Sans et al., 2006; Rodríguez \& González).

Por otra parte, se impone puntualizar que existe una exigua cuantía de habitantes de origen asiático, en general, oriundos de Japón, China, y Corea.

Con respecto al Brasil, es dable señalar que, en líneas generales, siguió los mismos parámetros de Uruguay, aunque con algunas peculiaridades vernáculas. De hecho, el propio descubrimiento por parte de los portugueses, trajo consigo el primer contacto directo con los grupos indígenas nativos y, consecuentemente, marcó el inicio de un paulatino y natural proceso de mestizaje (Knepper et al., 2011).

En la segunda mitad del siglo XVI, se produjo la llegada de los primeros esclavos africanos que se mantuvo por más de trescientos años, lo que llevó a que cerca de cuatro millones de individuos arribaran al país, y que actualmente, sus descendientes representen el $46 \%$ de la población del mismo. Asimismo, durante todo el período colonial, se sucedieron numerosas incursiones francesas, inglesas, holandesas y españolas, a diferentes regiones de su territorio (Ferreira; Heringer; Torres; Prado \& Caria).

Iniciada la segunda mitad del siglo XIX, Brasil recibió un nuevo y significativo contingente migratorio, procedente de casi toda Europa (fundamentalmente de Italia), Medio Oriente y Asia (Ferreira; Heringer; Torres; Knepper et al.).

Con la llegada de los primeros inmigrantes japoneses, en junio de 1908, se dio inicio a la llamada "historia nikkei brasileña", quienes se radicaron en São Paulo y alrededores, conformándose así la mayor colonia nipona que existe en el orbe (Kikumura-Yano, 2002).
De todo lo reseñado se desprende, que no existe un estereotipo físico o genético del ciudadano brasileño, dado que éste no es más que la síntesis de una larga, compleja y riquísima historia etnocultural (Florentino \& Machado, 2002).

Teniendo en cuenta los aspectos antedichos, es comprensible que no se hallasen discrepancias, en lo referente a las dimensiones del ancho mandibular, altura de la sínfisis y ancho bicoronoideo, entre las mandíbulas uruguayas $(37,65$ $\mathrm{mm} \pm 3,56 \mathrm{~mm}, 28,18 \mathrm{~mm} \pm 3,63 \mathrm{~mm}$ y $95,69 \mathrm{~mm} \pm 6,28$ $\mathrm{mm})$ y las brasileñas $(36,93 \mathrm{~mm} \pm 3,17 \mathrm{~mm}, 29,36 \mathrm{~mm} \pm$ $4,48 \mathrm{~mm}$ y $94,71 \mathrm{~mm} \pm 6,11 \mathrm{~mm}$, respectivamente). En base a idénticos criterios, podría inferirse que las variaciones morfológicas existentes entre los grupos observados, serían el corolario de los diversos factores raciales y étnicos que tomaron parte en la conformación de las actuales poblaciones de los países en cuestión (Sans et al., 1997; Ferreira; Heringer; Torres; Hidalgo et al.; Sans et al., 2006).

Claro está que otros agentes tendrían también la posibilidad de inducir efectos similares, como es el caso de la musculatura esquelética involucrada en las numerosas funciones llevadas a cabo en esta región, la que desempeña un preponderante rol, en cuanto al crecimiento, desarrollo y determinación de los patrones de remodelado óseo de todas sus estructuras. En realidad, la ejecución y mantenimiento de movimientos y funciones equilibradas constituyen el principal factor de crecimiento mandibular, ya que la mandíbula, hueso impar y único móvil de la cara, crece por proliferación endocondral a nivel condilar (hacia atrás, arriba y lateral, a través del cartílago secundario que rodea la cabeza del cóndilo), y por aposición y reabsorción ósea superficial en los sectores anteriores y posteriores de sus respectivas ramas. Dicho cartílago posee la capacidad de adecuarse a los requerimientos funcionales y a la dinámica masticatoria, a tal extremo que la simple puesta en actividad del músculo pterigoideo lateral modela, incentiva o limita su rango de crecimiento, modificando inclusive la dirección de éste. Algo similar ocurre con la forma y volumen del proceso coronoideo, los cuales se encuentran directamente condicionados a las características morfofuncionales del músculo temporal (Moss \& Salentijn, 1969; Enlow, 1992; Hunt; Humphrey et al.; Jiménez et al., 2006; Bolini et al.; Röhrle \& Pullan, 2007; Rai et al.). Siguiendo esta línea de razonamiento, se tornaría viable justificar los motivos que determinaron que las mandíbulas uruguayas poseyeran mayor profundidad de la incisura $(13,82 \mathrm{~mm} \pm 2,08 \mathrm{~mm})$, grosor del cuerpo $(13,52 \mathrm{~mm} \pm 1,77 \mathrm{~mm})$ y longitud del cóndilo $(20,09 \mathrm{~mm} \pm 2,40 \mathrm{~mm})$ que las brasileñas $(13,09 \mathrm{~mm} \pm$ $2,03 \mathrm{~mm}, 11,00 \mathrm{~mm} \pm 2,16 \mathrm{~mm}$ y $18,39 \mathrm{~mm} \pm 2,41 \mathrm{~mm}$, respectivamente). 
Igualmente, las mandíbulas uruguayas exhibieron guarismos superiores a los de las brasileñas $(34,68 \mathrm{~mm} \pm$ $3,04 \mathrm{~mm}$ contra $32,91 \mathrm{~mm} \pm 3,64 \mathrm{~mm}$ ), respecto a la distancia entre el proceso coronoideo y el cóndilo. Tal situación podría deberse a la estrecha correspondencia que se revela entre el biotipo y las particularidades anatomofuncionales del sistema neuromuscular facial, la que básicamente se pone en evidencia en presencia de severos cuadros de desarmonía o desequilibrio funcional (Hylander, 1977, 1979; Radu et al., 2004; Rai et al.).

Por otro lado, innúmeras investigaciones han sugerido el empleo de registros o análisis electromiográficos y/o modelos dinámicos tridimensionales, con el objetivo de estudiar en detalle los principios biomecánicos que rigen las diferentes funciones del sistema estomatognático y probar que los movimientos de la mandíbula durante la masticación, forman parte de una cadena cinética, en conjunto con las oscilaciones rítmicas y armónicas del hiodes, lengua y paladar blando, siendo consecuencia de la acción coordinada, sinérgica o antagonista, de diversos grupos musculares (craneomandibulares o masticadores clásicos, supra e infrahiodeos, entre otros). Por tanto, algunas de las divergencias evidenciadas entre las mandíbulas de las dos muestras examinadas, podrían ser el resultado del tipo, incidencia y magnitud de las fuerzas masticatorias ejercidas y de las consecuentes y sucesivas adaptaciones o compensaciones biológicas del propio sistema (MacDonald \& Hannam, 1984; Kaifu; Jiménez et al.; Röhrle \& Pullan; Hannam et al., 2008; Scott et al., 2009; Grine et al., 2010; Zaffaroni Piaggio \& Fiorestti).

No se verificaron apreciables discrepancias entre mandíbulas de uruguayos y brasileños en el ancho mandibular $(37,65 \mathrm{~mm} \pm 3,56 \mathrm{~mm}$ y $36,93 \mathrm{~mm} \pm 3,17 \mathrm{~mm})$, a nivel del primer molar, y a la altura de la sínfisis $(28,18$ $\mathrm{mm} \pm 3,63 \mathrm{~mm}$ y $29,36 \mathrm{~mm} \pm 4,48 \mathrm{~mm}$, respectivamente) Estas particularidades podrían deberse a la influencia de los variados factores sistémicos y al criterio adoptado para la selección de las correspondientes piezas anatómicas (mandíbulas dentadas). Efectivamente, si bien se sabe que cada grupo humano o sujeto puede presentar una morfología craneofacial específica y disímil de la de sus congéneres, es lógico esperar una tendencia similar en presencia de condiciones análogas. La fuerza masticatoria, producida por los cortos y potentes músculos de la región, con punto de aplicación en el área molar y resultante en el de la sínfisis, se constituirá así en un auténtico elemento modelador del hueso, tanto en el sector posterior como en el anterior de la mandíbula. Naturalmente que ante un mayor número de contactos dentarios, sean estos funcionales o parafuncionales, existirá un incremento generalizado de la actividad muscular y, por consiguiente, de la potencia o fuerza empleada, lo que generará un mayor grado de aposición y consolidación ósea en las áreas antedichas. Este activo y cambiante proceso biomecánico se acompasará además, en condiciones fisiológicas, con el crecimiento del hueso alveolar compensatorio de la continua erupción dentaria, recordando que el mismo existe en función de los diversos dientes e involuciona ante la eventual pérdida de ellos (Hylander, 1984; MacDonald \& Hannam; Planas, 1987; Sasaki et al., 1989; Kaifu; Bolini et al.; Antón et al.).

En conclusión, las mediciones en las mandíbulas de individuos uruguayos dieron resultados notoria o levemente superiores, con excepción de las correspondientes a la altura de la sínfisis, ligeramente mayores en sus homólogas brasileñas. Las diferencias más expresivas entre los dos grupos estudiados, correspondieron a la altura de la incisura, altura de la rama y longitud mandibular, en tanto que el menor grado de discrepancia, se evidenció en el ancho mandibular, altura de la sínfisis y ancho bicoronoideo.

SASSI, C.; PICAPEDRA, A.; CARIA, P. H. F.; GROPPO, F.; FRANCESQUINI JÚNIOR, L. ; DARUGE JÚNIOR, E. \& PRADO F. B. Anthropometric comparison between mandibles from Uruguayan and Brazilian populations. Int. J. Morphol., 30(2):379-387, 2012.

SUMMARY: The human mandibles belonging to Uruguayan population present some anatomic differences with those belonging to Brazilian population. This appears to be determined by racial and ethnic characteristics of each country. This study examined the morphological characteristics of 100 Uruguayan mandibles and compared them with similar number of Brazilian bones, using thirteen measurements stipulated by Humphrey et al. (1999). The significant variations in mean values were calculated by Mann-Whitney test $($ alpha $=5 \%)$. Expressive discrepancies were observed between the two populations regarding some of analyzed dimensions. The Uruguayan mandibles showed higher values in almost all measurements when compared to the Brazilian ones, except for the mandibular symphysis height, which was lightly higher in Brazilian mandibles. Differences were observed in notch and ramus height, mandibular length, mandibular width, symphysis height and bicoronoid width. Several factors could be responsible for these morphological discrepancies, including functional and biomechanical adaptations of stomatognathic system, stimulated by different consistency of the diet or miscegenation.

KEY WORDS: Mandible; Morphology; Anthropometry; Brazil; Uruguay. 


\section{REFERENCIAS BIBLIOGRÁFICAS}

Anderson, J. Y. Mandibular Morphology in human populations: a examination of a primary muscle attachment and architectonic models for development of the ramus. Am. J. Phys. Anthropol., 26:64,1998.

Antón, S. C.; Carter-Menn, H. \& DeLeon, V. B. Modern human origins: continuity, replacement, and masticatory robusticity in Australasia. J. Hum. Evol., 60(1):70-82, 2011.

Bolini, P. D. A.; Gaspar, M. A. M. \& De Souza, J. F. Dimensión Vertical de la Región Anterior de la Mandíbula. Int. J. Morphol., 24(4):531-3, 2006.

Bogin, B. \& Rios, L. Rapid morphological change in living humans: implications for modern human origins. Сomp. Biochem. Physiol. A Mol. Integr. Physiol., 136(1):71-84, 2003.

Enlow, D. Crecimiento Maxilo Facial. $3^{\text {a }}$ ed. Pennsylvania, Ed. McGraw-Hill, 1992.

Ferreira, R. F. The construction of the afro-descendant identity: contributions of brazilian psychology. Interacões Estud. Pesq. Psicol., 3(5):93-108, 1998.

Florentino, M. \& Machado, C. Ensaio sobre a imigração portuguesa e os padrões de miscigenação no Brasil (séculos XIX e XX). PSR, 10(1):58-84, 2002.

Grine, F. E.; Judex, S.; Daegling, D. J.; Ozcivici, E.; Ungar, P. S.; Teaford, M. F.; Sponheimer, M.; Scott, J.; Scott, R. S. \& Walker, A. Craniofacial biomechanics and functional and dietary inferences in hominin paleontology. J. Hum. Evol., 58(4):293-308, 2010.

Hannam, A. G.; Stavness, I.; Lloyd, J. E. \& Fels, S. A dynamic model of jaw and hyoid biomechanics during chewing. $J$. Biomech., 41(5):1069-76, 2008.

Heringer, R. Racial inequalities in Brazil: a synthesis of social indicators and challenges for public policies. Cad. Saude Publica., 18:57-65, 2002.

Hidalgo, P. C.; Bengochea, M.; Abilleira, D.; Cabrera, A. \& Alvarez, I. Genetic Admixture Estimate in the Uruguayan Population Based on the Loci LDLR, GYPA, HBGG, GC and D7S8. Int. J. Hum. Genet., 5(3):217-22, 2005.

Humphrey, L. T.; Dean, M. C. \& Stringer, C. B. Morphological variation in great ape and modern human mandibles. $J$. Anat., 195 ( Pt 4):491-513, 1999.

Hunt, N. Muscle function and the control of facial form. In: Harris, M.; Edgard, M. \& Meghji, S. (Eds.). Clinical Oral Science. Oxford, Wrigth, 1998.
Hylander, W. L. The adaptive significance of Eskimo craniofacial morphology. In: Dahlberg, A. A. \& Graber, T. M. (Eds.). Orofacial Growth and Development. Paris, Mouton, 1977.

Hylander, W. L. The functional significance of primate mandibular form. J. Morphol., 160(2):223-40, 1979.

Hylander, W. L. Stress and strain in the mandibular symphysis of primates: a test of competing hypotheses. Am. J. Phys. Anthropol., 64(1):1-46, 1984.

Jiménez, V. M.; González, E. V. \& Contasti, G.Influencia de la mordida cruzada posterior unilateral en el crecimiento mandibular. Rev. Latinoam. Ortod. Odontoped., 2006. Disponible en: http://www.ortodoncia.ws/publicaciones/2006/ mordida_cruzada_posterior_unilateral.asp

Kaifu, Y. Changes in mandibular morphology from the Jomon to modern periods in eastern Japan. Am. J. Phys. Anthropol., 104(2):227-43, 1997.

Kikumura-Yano, A. Encyclopedia of Japanese Descendants in the Americas: An Illustred History of the Nikkei. Walnut Creek, CA, AltaMira Press, 2002. p.115.

Knepper, C.; Cipriano, W.; Esgalha, S. V. \& Waqued Neto, R. Embajada de Brasil. Bogotá - Colombia. 2011. Disponible en: http://www.brasil.org.co/htm/index.htm

Lieberman, D. E.; Krovitz, G. E.; Yates, F. W.; Devlin, M. \& St Claire, M. Effects of food processing on masticatory strain and craniofacial growth in a retrognathic face. J. Hum. Evol., 46(6):655-77, 2004.

MacDonald, J. W. \& Hannam, A. G. Relationship between occlusal contacts and jaw-closing muscle activity during tooth clenching: Part I. J. Prosthet. Dent., 52(5):718-28, 1984.

Moss, M. L. \& Salentijn, L. The primary role of the functional matrix in facial growth. Am. J. Orthod., 55(6):566-77, 1969.

Planas, P. Rehabilitación Neuroclusal (RNO). Barcelona, Salvat, 1987.

Prado, F. B. \& Caria, P. H. F. Comparaciones morfológicas entre las mandíbulas de brasileños y de poblaciones de otros continentes. Int J. Morphol., 25(2):323-7, 2007.

Radu, M.; Marandici, M. \& Hottel, T. L. The effect of clenching on condylar position: A vector analysis model. J. Prosthet. Dent., 91(2):171-9, 2004.

Rai, R.; Ranade, A. V.; Prabhu, L. V.; Pai, M. M.; Madhyastha, S. \& Kumaran, M. A Pilot Study of the Mandibular Angle and Ramus in Indian Population. Int. J. Morphol., 25(2):353-6, 2007. 
Rodríguez, S. \& González, R. En busca de los orígenes perdidos. Los guaraníes en la construcción del ser uruguayo. Montevideo, Planeta, 2010. p.532.

Röhrle, O. \& Pullan, A. J. Three-dimensional finite element modelling of muscle forces during mastication. J. Biomech., 40(15):3363-72, 2007.

Sanguinet, L. Breve historia de las migraciones. 2001. Disponible en: http://www.anticapitalistas.org/espacio/campa/ uruguay.htm

Sans, M.; Salzano, F. M. \& Chakraborty, R. Historical genetics in Uruguay: estimates of biological origins and their problems. Hum. Biol., 69(2):161-70, 1997.

Sans, M. Admixture studies in Latin America: from the 20th to the 21st century. Hum. Biol., 72(1):155-77, 2000.

Sans, M.; Merriwether, D. A.; Hidalgo, P. C.; Bentancor, N.; Weimer, T. A.; Franco, M. H.; Alvarez, I.; Kemp, B. M. \& Salzano, F. M. Population structure and admixture in Cerro Largo, Uruguay, based on blood markers and mitochondrial DNA polymorphisms. Am. J. Hum. Biol., 18(4):513-24, 2006.

Sasaki, K.; Hannam, A. G. \& Wood, W. W. Relationships between the size, position, and angulation of human jaw muscles and unilateral first molar bite force. J. Dent. Res., 68(3):499-503, 1989.

Scott, J. R.; Godfrey, L. R.; Jungers, W. L.; Scott, R. S.; Simons, E. L.; Teaford, M. F.; Ungar, P. S. \& Walker, A. Dental microwear texture analysis of two families of subfossil lemurs from Madagascar. J. Hum. Evol., 56(4):405-16, 2009.

Suazo, G. I. C.; Zavando, M. D. A. \& Smith, R. L. Blind test of mandibular morphology with sex indicator in subadult mandibles. Int. J. Morphol., 26(4):845-8, 2008.

Torres, C. People of African descent in the region of the Americas and health equity. Rev. Panam. Salud Publica, 11(5-6):471-9, 2002.

Wood, B. A.; Li, Y. \& Willoughby, C. Intraspecific variation and sexual dimorphism in cranial and dental variables among higher primates and their bearing on the hominid fossil record. J. Anat., 174:185-205, 1991.

Zaffaroni Piaggio, A. \& Fiorestti, H. Influencia de las funciones y parafunciones en el crecimiento y desarrollo craneofacial. Actas Odontol., 7(1):15-30, 2010.

\author{
Dirección para correspondencia: \\ Dr. Carlos Sassi. \\ Departamento de Odontologia Legal e Deontologia \\ Área de Odontologia Social \\ Faculdade de Odontologia de Piracicaba - FOP/UNICAMP \\ Avenida Limeira, 901 - Bairro Areião. \\ CEP 13414-903, Piracicaba / SP \\ BRASIL
}

Email: almapipa@adinet.com.uy

Recibido : 11-07-2011

Aceptado: 02-01-2012 